\title{
LONG-TERM TRENDS OF CHLAMYDIA TRACHOMATIS IN A CLINIC POPULATION AT THE ROYAL WOMENS HOSPITAL, MELBOURNE
}

Running Title:

30 years Chlamydia screening of women

GARLAND, Suzanne Marie 1,2,3,

Prof. Reproductive \& Neonatal Infectious Diseases,

Suzanne.Garland@,thewomens.org.au

SUBASINGHE Asvini Kokila ${ }^{1,3}$,

Project Officer

asvini.subasinghe@gmail.com

AHMED Navera ${ }^{1,2}$,

Medical student at the time of the study

naverasahmed@gmail.com

JAYASINGHE Yasmin ${ }^{1,2,4}$,

Consultant Gynaecologist - RSU and Oncology/Dysplasia

Gynaecology

yasmin.jayasinghe@unimelb.edu.au

MARCEGLIA Alex ${ }^{5}$

Unit Head $\mid$ Sexual Health and Rapid Access Service

alex.marceglia@thewomens.org.au

${ }^{1}$ Centre for Women's Infectious Diseases Research, The Royal Women's Hospital, Parkville, Australia

This is the author manuscript accepted for publication and has undergone full peer review but has not been through the copyediting, typesetting, pagination and proofreading process, which may lead to differences between this version and the Version of Record. Please cite this article as doi: $10.1111 / \mathrm{AJ} 0.13106$

This article is protected by copyright. All rights reserved 
${ }^{2}$ Department of Obstetrics and Gynaecology, Royal Women's Hospital, University of Melbourne, Parkville, Australia

${ }^{3}$ Infection \& Immunity Theme, Murdoch Children's Research Institute, Parkville, Australia

${ }^{4}$ Department of Gynaecology, Royal Children's Hospital, Parkville, Australia

${ }^{5}$ Sexual Health and Rapid Access Service, The Royal Women's Hospital, Parkville, Australia

\section{Corresponding Author:}

Suzanne M Garland

Royal Women's Hospital

Level 7

Locked Bag 300

Parkville VIC 3052

Tel: 83453670

suzanne.garland@thewomens.org.au

Acknowledgement:

We thank Alexandra Gorelik for sage comments on the statistics

\section{Conflict of Interest:}

Professor Suzanne Garland, has no conflict of interest with respect to the subject of this manuscript. Professor Suzanne Garland, has received from Merck through her institution for an investigator initiated grant to evaluate HPV vaccine effectiveness study in a young women's study, speaker fees from MSD for work performed in her personal time, and is a member of the Global Advisory Board for Merck. All other authors report no conflicts to report.

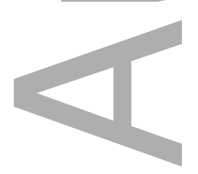


3

4

5 Article type : Original Manuscript

6

7

8

LONG-TERM TRENDS OF CHLAMYDIA TRACHOMATIS IN A CLINIC POPULATION AT THE ROYAL WOMENS HOSPITAL, MELBOURNE

10

30 years Chlamydia screening of women

12

Key Words: Chlamydia trachomatis, chlamydia, prevalence, assays

14

15 Main text word count: 1802

16 Abstract word count: 233

17 Figure count: 3

18 Table count: 0

19 References 27

20

21 Abstract

22 Background:

This article is protected by copyright. All rights reserved 
1 Chlamydia trachomatis (C. trachomatis) prevalence has been reported to be increasing.

2 Whether this is a true increase over time or confounded by increases in testing and/or use of 3 more sensitive assays is to be determined.

4 Materials and Methods:

5 One laboratory service has been detecting C. trachomatis for the past 30 years within the

6 Royal Women's Hospital Melbourne. We conducted a retrospective audit of records over the

7 period 1986 to 2016 from a clinic population routinely offered chlamydia screening. These

8 were women presenting for family planning advice (termination of pregnancy, intrauterine

9 device insertion or considered at high risk), who underwent chlamydia testing in the context of various diagnostic assays used over this time period. Assays utilized included culture, enzyme immunoassay (EIA), DNA probe, and nucleic acid amplification testing (NAAT).

Non parametric test for trend was used to determine significant differences between prevalence estimates across ordered groups. Least squares regression was conducted to describe a linear trend matching known data points.

\section{Results:}

Overall, there was no significant change for chlamydia prevalence which was $2.2 \%$, in the 30-year study period ( $\mathrm{p}=0.7$ ). Over time diagnostic assays changed from culture, to EIA, DNA probe, to the more sensitive NAAT. The bulk of the positives were in women under 25 years of age $(57 \%)$.

\section{Conclusion:}

Chlamydia prevalence has been stable over 30 years, remaining a problem in young women. Screening for those at risk needs underscoring in a national sexual health program.

233 words

\section{Introduction}

Chlamydia trachomatis is the most common, notifiable, sexually transmissible, bacterial infection in Australia ${ }^{1}$. Most often a silent infection, untreated it holds severe consequences in women, including infertility, pelvic inflammatory disease, ectopic pregnancy and chronic pelvic pain ${ }^{2}$. It disproportionately affects the young, with the majority occurring in those less than 25 years of age $e^{3,4}$.

Despite this, chlamydia only became notifiable in most States and Territories of Australia in 1991 and not until 1997 was it mandated in NSW ${ }^{5}$. Even then, notifications are reported as positive tests per 100,000 population without giving any indication of the numbers of tests 
1 performed as denominator data or type of assay, which may also contribute to rising 2 notifications. In 2016 there were 260,000 new cases of chlamydia notified in the total 3 Australian population ${ }^{6}$, whereas in 2011 , there were 79,833 compared to 20,266 in $2001^{7}$.

4 This is a staggering fourfold increase in just ten years and tenfold in 15 years. This rise has

5 been attributed to increased testing ${ }^{8,9}$, as well as an increase in the rate of positive test 6 results $^{10}$. To obtain a better handle on these national surveillance trends, a systematic review 7 and meta-analysis of reports from 1997 to 2011 evaluating numbers of tests performed within 8 clinical settings as the denominator, was undertaken by Lewis et al. ${ }^{4}$ They calculated the pooled preyalence of genital chlamydial infection in women aged 16-25 years attending general practice and those attending sexual health services to be $5.0 \%$ and $6.8 \%$ respectively ${ }^{4}$. This is in keeping with Victorian data from the comprehensively conducted Victorian Primary Care Network for Sentinel Surveillance, 2007-2011, which reported laboratory data from sexual health and primary care clinics ${ }^{11}$. Chlamydia positivity increased significantly over the study time from $5.1 \%$ to $6.3 \%$ for women, with particular increases in those aged 1624 years. These authors concluded that increased testing alone could not explain the increase in positive results over time ${ }^{11}$.

To address these inconsistences, of whether prevalence of chlamydia has truly increased or reports are confounded by increased testing and use of more sensitive assays, we reviewed data from a clinic population, where chlamydia screening has occurred over three decades and by the same Microbiology diagnostic department.

\section{Materials and Methods}

\section{Study population}

This was a retrospective audit of chlamydia screening in women attending the Royal Women's Hospital [RWH], Melbourne, for family planning advice from 1986 to 2016 and approved by the Royal Women's Hospital Research and Ethics Committees. Prior to the formation of the Choices clinic in 1998, two clinics named the Pregnancy Advisory clinic [PAS] and the Family Planning clinic (FPC) existed. Their amalgamation in 1998 resulted in the Choices clinic. The Choices Clinic conducts over 2000 consultations annually and provides a sexual health service, including chlamydia screening, contraception and family planning services to all patients including those undergoing termination of pregnancy (TOP). 
1 The number of patients seen in PAS/Choices clinic remained stable over the course of the 2 study.

3 The Medipath electronic database was introduced in 1995. Subjects attending from 1995 4 were identified and results obtained via Medipath. Due to the variability in the combination 5 of clinic names, multiple searches on the following items were undertaken: "Choices", 6 "Choices-FPC", "Choices- PAS", "FPC", "PAS". Data prior to April 1995, from 1986 was 7 manually recorded by one of us (SMG), as part of an ongoing audit. Data was deidentified, with each subject given a unique code for the purposes of this audit. Ethics approval for this audit was obtained by the Royal Women's Hospital Human Research and Ethics Committees.

\section{Chlamydia trachomatis testing}

The Microbiology Department at RWH has been conducting diagnostic assays for C. trachomatis since the mid-1980s, when culture techniques were developed ${ }^{12}$. Routine screening for C. trachomatis began in 1988 after a prevalence study of different patient populations found the highest rates in those presenting for surgical TOP (at around $4 \%)^{12}$. This was underscored by those women with $\mathrm{C}$ trachomatis having an increased risk of postabortal sepsis ${ }^{13}$. Since that time, testing methods have evolved from culture to rapid antigen based assays of enzyme immunoassay (EIA), direct immunofluorescence (DFT) ${ }^{14,15}$ to DNA probe ${ }^{16}$ to nucleic acid amplification testing (NAAT) specifically, polymerase chain reaction (PCR) ${ }^{17}$, the assay used to date ${ }^{17,18}$. Specimens consisted of clinician collected endocervical swabs for culture, EIA, DFT, DNA probe, whilst for PCR tests high vaginal swabs or urine specimens were collected and validated as appropriate ${ }^{19}$.

\section{Statistical analysis}

Denominator data captured was all tests for chlamydia undertaken for female patients attending Choices [or equivalent clinic], between the years 1986 to 2016. Numerator data were defined as all positive test results for chlamydia, whatever the assay. The records included basic demographic information such as age, sex and postcode.

As part of the Choices Clinic protocol, patients who receive a positive result are required to undergo repeat testing in 6 weeks from treatment, to confirm cure of the infection. Patients attending for follow-up testing were identified using their unique code and the dates between testing. Follow-up tests were defined as tests taken less than 60 days after the initial test and were excluded from analysis; such that duplicate and triplicate patient identifier, which were 
recorded less than 60 days after initial testing were excluded from analyses. Tests undertaken for males and neonates were also excluded.

The data obtained were analysed using STATA version 15 (College Station, Texas) ${ }^{20}$. Non parametric test for trend was used to determine significant differences between prevalence estimates across ordered groups. Least squares regression was conducted to describe a linear trend matching known data points.

(
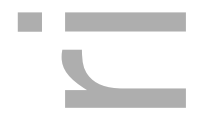

\section{Results}

The prevalence of chlamydia over time per assay type from 1986 to 2016 is shown in Figure 1. It is to be noted that there are overlap periods when more than one assay was being utilised: for example, whilst culture and PCR was being introduced in 1996-1997 and again 2001 when LCR and PCR was being introduced. Even during the period when PCR was used exclusively from 2002 to today, there is fluctuation, although no significant change. Allowing for this variability for the various assays (see dotted line), a constant trend over time is apparent, at a prevalence of $2.2 \%$. Specifically, for women aged 17-24, it stayed relatively constant $(\sim 4.5 \%)$. There is no significant change in positivity over the 30-year time period time $(\mathrm{P}=0.7)$.

Whilst the mean age of women was 29 years, as shown in Figure 2, the bulk of positives were detected for women under the age of 25 years (57\%, with $86 \%$ being younger than 34 years of age.

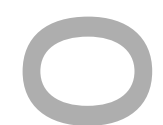

Figure 3 shows positivity as a function of the different assay types for the time period for which each were utilised. DNA probe showed the lowest rate of positivity of 2.4 , followed by culture at $2.7 \%$ and PCR at $3.3 \%$. Positivity was shown to be greater by PCR compared DNA probe $(\mathrm{P}<0.001)$, but not compared with culture.

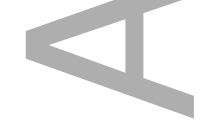

\section{Discussion}

In this large audit over three decades, utilising the same laboratory to measure the prevalence 
of chlamydia within a similar patient population, those presenting largely for termination of pregnancy, or high risk family planning advice, a relatively high, but constant rate is shown $(2.2 \%, p=0.7)$. The fact that with time from 2002 to 2016 shows a trend (but not statistically significant) to increase likely reflects an increase in the sampling population and utilising assays with increased sensitivity. As found in most studies the majority of chlamydia cases were found in younger women. The rate we describe is not dissimilar to that described in the Australian Chlamydia Control Effectiveness Pilot - ACCEPt study, which was conducted between 2010 and 2011, where the prevalence of chlamydia was reported to be $4.6 \%$ overall and $4.4 \%$ in women ${ }^{21}$. Despite guidelines recommending screening ${ }^{22}$ of those $<29$ years of age, recent change of partner, and other risk behaviours, these guidelines have been slow to be implemented. On a national scale in $2015^{23}$, only $12 \%$, a low proportion of young people aged $15-29$ years were tested for chlamydia ${ }^{23,}$ although this did represent a relative increase of $69 \%$ since $2008^{24}$.

In an examination of laboratory records available from general practitioner clinics and the Canberra Sexual Health Centre, Currie and Bowden indicated that while the number of tests had increased by $48.2 \%$ between 1998 and 2004 in the Australian Capital Territory, the proportion of positive tests had also risen by $37.8 \%$ from $3.7 \%$ to $5.1 \%^{22}$. This data is also supported by an analysis of Medicare testing data by Chen et al., demonstrating that the chlamydia test positivity rate for women increased from $4.3 \%$ in 1999 to $7.3 \%$ in $2001^{25}$. A limitation of both aforementioned studies is that they do not refer to the type of diagnostic test performed, and thus the sensitivity of the test results is not known. Conversely, from 2008 to 2015, the ratio of chlamydia notifications to chlamydia tests funded by Medicare declined in $15-29$ years olds by $3.6 \%$ from 13.5 notifications per 100 tests in 2008 to 9.9 notifications per 100 tests in $2015^{24}$.

Whilst the strength of this study being conducted in the one laboratory service over three decades, a limitation is not being able to define those who were presenting for TOP in the Choices clinic, once combined with Family Planning. Those presenting for TOP are a higher risk population. Unpublished data from one of us (AM) from separate audits during the time that PAS (women presenting for TOP) and FPC (for women presenting for contraception advice) could be identified independently within Choices, revealed that the former group had higher rates (Aug 2006 to Aug 20071183 PAS women had a chlamydia positive rate of $4.1 \%$, whereas for 983 FPC women it was $1.6 \%$ : similarly in a 2000/2001 audit chlamydia 
1 positivity for the two clinics was $3.5 \%$ vs $1 \%$ respectively. In an audit of 1636 PAS patients

$21 / 8 / 2009$ to 31/12/2010 chlamydia positivity was 5.3\%). This underscores the importance of screening all women presenting for TOP. Thus the overall figure of $2.2 \%$ is a combination of the higher risk PAS patients and the lower risk FPC patients.

It is noteworthy that diagnosis and appropriate treatment for chlamydia infection, (including simultaneous treatment of the partner) particularly in the setting of a surgical procedure is paramount, as reinfection of chlamydia is not uncommon. In Walker's study in General

Practice the risk of reinfection was $20 \%$ in the first year, with $50 \%$ of reinfections occurring between 3.5 and 6.6 months $^{26}$. Hence, this is the reason we recommend a test of reinfection at 3 months for uncomplicated chlamydia management. Moreover, since the switch to monotherapy with azithromycin treatment [this went onto the TGA register in 1994], rather than 10 days of doxycycline treatment, incomplete treatment is less of a problem. Then again within the sexual health setting of screening for pathogens at risk of becoming upper tract complications following a surgical procedure, is the matter of macrolide resistance to Mycoplasma genitalium where resistance to azithromycin has risen to $50 \%$ or more ${ }^{27}$. Much more work is to be done to impact chlamydia rates in young people, but as rates have remained unchanged, a major focus on testing and treating chlamydia to reduce its harmful impacts remains an important focus of care. This is particularly important with the implementation of delayed cervical screening protocols internationally, reducing opportunities for preventative sexual health screening. Lessons may be learned from the cervical screening and HPV vaccination public awareness campaigns which destigmatised HPV and facilitated active participation in health behaviours.

1. Currie MJ, Bowden FJ. The importance of chlamydial infections in obstetrics and

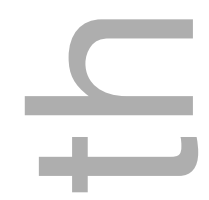
gynaecology: an update. Aust N Z J Obstet Gynaecol. Feb 2007;47(1):2-8.

2. Goller JL, De Livera AM, Fairley CK, et al. Population attributable fraction of pelvic inflammatory disease associated with chlamydia and gonorrhoea: a cross-sectional analysis of Australian sexual health clinic data. Sex Transm Infect. Apr 182016.

3. Garland SM, Johnson B. Chlamydia trachomatis infections--the Royal Women's Hospital experience. Med J Aust. Feb 20 1989;150(4):174-177. 
4. Lewis D, Newton DC, Guy RJ, et al. The prevalence of Chlamydia trachomatis infection in Australia: a systematic review and meta-analysis. BMC Infectious Diseases. May 14 2012;12(1):113.

5. Newman L, Stirzaker S, Knuckey D, et al. Australia's notifiable disease status, 2008: annual report of the National Notifiable Diseases Surveillance System. Communicable diseases intelligence quarterly report. 2010/09// 2010;34(3):157-224.

6. Kirby Institute. Bloodborne viral and sexually transmissible infections in Aboriginal and Torres Strait Islander people: annual surveillance report 2017.

https://kirby.unsw.edu.au/sites/default/files/kirby/report/KirbyInst_Indigenous_ASR2017compressed.pdf.

7. System NNDS. ABS Australian Demographic Statistics, (cat. no. 3101.0)2011.

8. Chen MY, Donovan B. Changes in testing methods for genital Chlamydia trachomatis in New South Wales, Australia, 1999 to 2002. Sexual health. 2005;2(4):251-253.

9. Chen MY, Donovan B. Genital Chlamydia trachomatis infection in Australia: epidemiology and clinical implications. Sexual health. 2004;1(4):189-196.

10. Ali $\mathrm{H}, \mathrm{Cameron} \mathrm{E}$, Drovandi $\mathrm{CC}$, et al. A new approach to estimating trends in chlamydia incidence. Sexually Transmitted Infections. 2015.

11. Lim MSC, El-Hayek C, Goller JL, et al. Trends in chlamydia positivity among heterosexual patients from the Victorian Primary Care Network for Sentinel Surveillance, 2007-2011. Med J Aust. 2014;200(3):166-169.

12. Lees MI, Newnan DM, Garland SM. Simplified culture procedure for large-scale screening for Chlamydia trachomatis infections. J Clin Microbiol. Jul 1988;26(7):1428-1430.

13. Wein P, Kloss M, Garland SM. Postabortal pelvic sepsis in association with Chlamydia trachomatis. Aust N Z J Obstet Gynaecol. Nov 1990;30(4):347-350.

14. Lees MI ND, Garland SM. Detection of Chlamydia trachomatis by direct immunofluorescence and culture in genital specimens of symptomatic and asymptomatic women. Venereology. 1990;3(2):28-31.

15. Lees M, Newnan D, Garland S. Detection of Chlamydia trachomatis by direct immunofluorescence and culture in genital specimens of symptomatic and asymptomatic women. Venereology. 1990;3:28-31.

16. Lees MI, Newnan DM, Garland SM. Comparison of a DNA probe assay with culture for the detection of Chlamydia trachomatis. J Med Microbiol. Sep 1991;35(3):159-161.

17. Tabrizi SN, Lees MI, Garland SM. Comparison of polymerase chain reaction and culture techniques for detection of Chlamydia trachomatis. Mol Cell Probes. Oct 1993;7(5):357-360. 
18. Tabrizi S, Chen S, Fairley C, et al. Tampon-collected genital cells in the detection of Chlamydia trachomatis by polymerase chain reaction. J Infect Dis. Sep 1993;168(3):796-797.

19. Garland SM, Tabrizi S, Hallo J, Chen S. Assessment of Chlamydia trachomatis prevalence by PCR and LCR in women presenting for termination of pregnancy. Sexually Transmitted Infections. 2000;76(3):173.

20. Bacon J, Francoeur D, Goldfarb AF, Breech LL. Abnormal pap smears in adolescents. [Review] [0 refs]. Journal of Pediatric \& Adolescent Gynecology. 2003;16(3):157-166.

21. Hocking JS, Spark S, Guy R, et al. 08 The Australian chlamydia control effectiveness pilot (ACCEPt): first results from a randomised trial of annual chlamydia screening in general practice. Sexually Transmitted Infections. 2012;88(Suppl 1):A3-A4.

22. Currie MJ, Bowden FJ. An epidemic of infection, not just testing: Chlamydia prevalence estimates in the Australian Capital Territory 1998-2004. Australian and New Zealand Journal of Public Health. 2006;30(3):286-287.

23. Bacon J. Management quandry. HPV in teenagers. Journal of Pediatric \& Adolescent Gynecology. 2003;16(3):147-148.

24. Kahn JA, Goodman E, Huang B, Slap GB, Emans SJ. Predictors of Papanicolaou smear return in a hospital-based adolescent and young adult clinic. Obstetrics \& Gynecology. 2003;101(3):490-499.

25. Chen MY, Fairley CK, Donovan B. Nowhere near the point of diminishing returns: correlations between chlamydia testing and notification rates in New South Wales. Australian and New Zealand Journal of Public Health. 2005;29(3):249-253.

26. Walker J, Tabrizi SN, Fairley CK, et al. Chlamydia trachomatis Incidence and Re-Infection among Young Women - Behavioural and Microbiological Characteristics. PLOS ONE. 2012;7(5):e37778.

27. Read TRH, Jensen JS, Fairley CK, et al. Use of Pristinamycin for Macrolide-Resistant Mycoplasma genitalium Infection. Emerging Infectious Diseases. 2018;24(2):328-335.
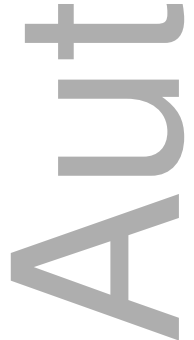

This article is protected by copyright. All rights reserved 


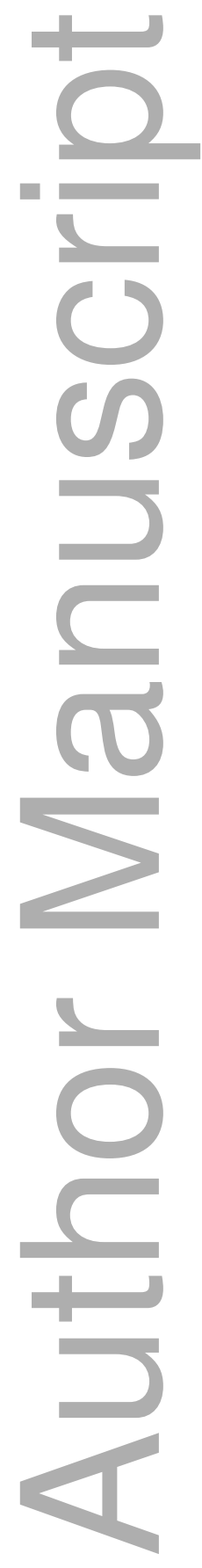

This article is protected by copyright. All rights reserved 


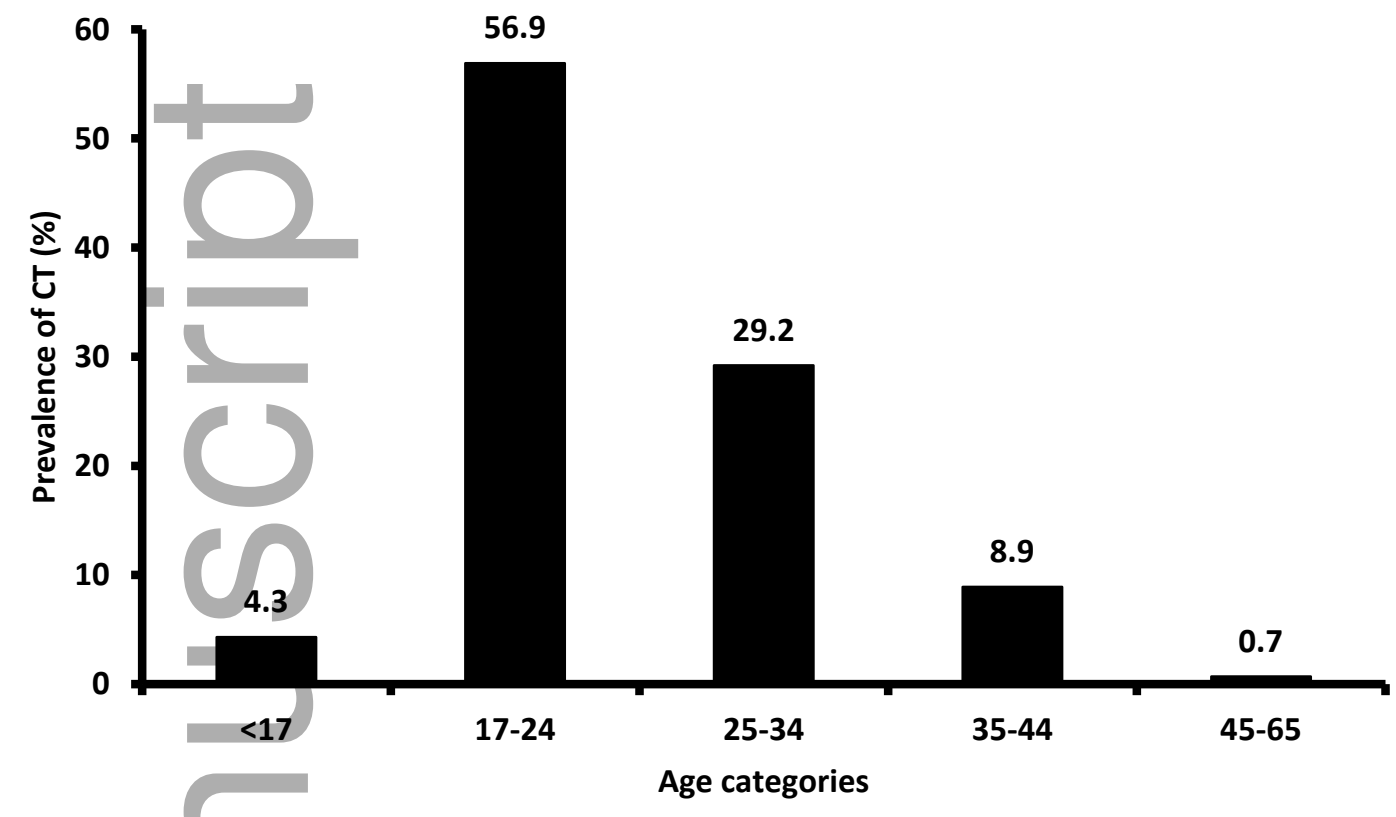

Figure 2. Prevalence of chlamydia by age category in women presenting at Choices Clinic, Royal Women's Hospital between 1995-2016 ( $\mathrm{n}=38,534)$

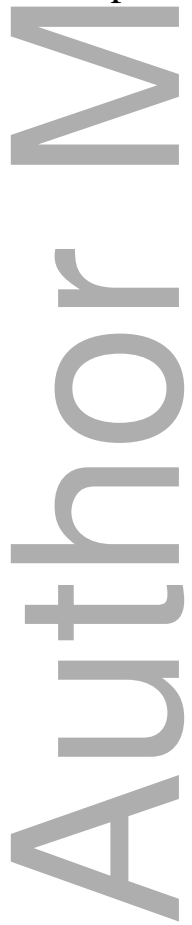

This article is protected by copyright. All rights reserved 


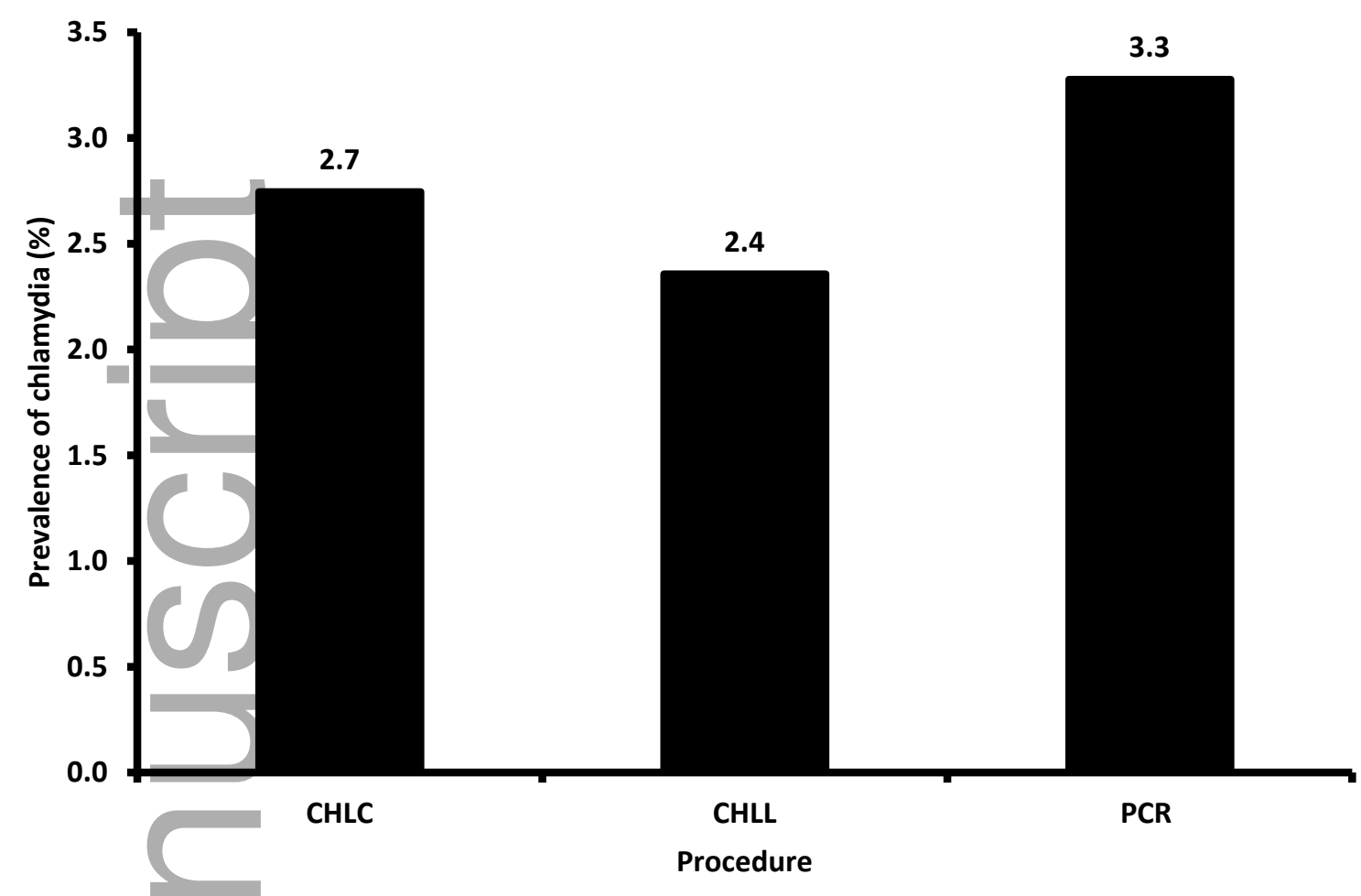

Figure 3. Prevalence of chlamydia over the study time period based on laboratory testing procedure, whereby CHLC is culture, CHLL DNA probe and PCR as polymerase chain reaction.

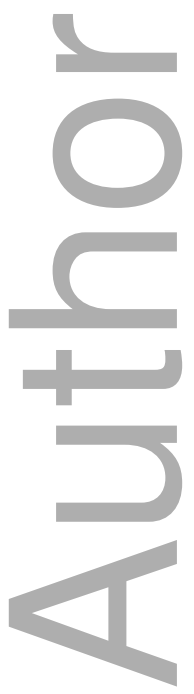




\section{University Library}

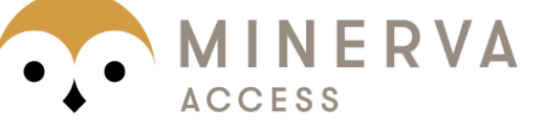

A gateway to Melbourne's research publications

Minerva Access is the Institutional Repository of The University of Melbourne

Author/s:

Garland, SM;Subasinghe, AK;Ahmed, N;Jayasinghe, Y;Marceglia, A

Title:

Long-term trends of Chlamydia trachomatis in a clinic population at the Royal Women's Hospital, Melbourne

Date:

2019-12-27

\section{Citation:}

Garland, S. M., Subasinghe, A. K., Ahmed, N., Jayasinghe, Y. \& Marceglia, A. (2019). Long-term trends of Chlamydia trachomatis in a clinic population at the Royal Women's Hospital, Melbourne. AUSTRALIAN \& NEW ZEALAND JOURNAL OF OBSTETRICS \& GYNAECOLOGY, 60 (1), pp.149-153. https://doi.org/10.1111/ajo.13106.

Persistent Link:

http://hdl.handle.net/11343/286788 